\title{
http://dx.doi.org/10.35381/racji.v5i8.585
}

\section{La vulneración de derechos del presunto agresor en la violencia intrafamiliar}

\section{Violation of the rights of the alleged aggressor in domestic violence}

\author{
Judith Amparo Valdez-Masache \\ judith.valdez@psg.ucacue.edu.ec \\ Universidad Católica de Cuenca, Cuenca \\ Ecuador \\ https://orcid.org/0000-0001-6719-9534 \\ Cecilia Ivonne Narváez-Zurita \\ inarvaez@ucacue.edu.ec \\ Universidad Católica de Cuenca, Cuenca \\ Ecuador \\ https://orcid.org/0000-0002-7437-9880 \\ Diego Fernando Trelles-Vicuña \\ dtrelles@ucacue.edu.ec \\ Universidad Católica de Cuenca, Cuenca \\ Ecuador \\ https://orcid.org/0000-0002-8466-7165 \\ Juan Carlos Erazo-Álvarez \\ jcerazo@ucacue.edu.ec \\ Universidad Católica de Cuenca, Cuenca \\ Ecuador \\ https://orcid.org/0000-0001-6480-2270
}

Recibido: 14 de noviembre de 2019

Aprobado: 15 de diciembre de 2019

\section{RESUMEN}

La violencia intrafamiliar como problema social, legal y de salud pública, vulnera los derechos del presunto agresor generando en sí consecuencias legales, transgrediendo derechos constitucionalmente reconocidos, así como el principio de inocencia. La legislación ecuatoriana ha establecido medios de defensa conducentes para evitar el cometimiento de actos de provocación ya que el propósito es impedir que el presunto culpable y su víctima se relacionen, además el análisis médico del estado de salud 
mental es ineludible para con ello conocer si existen anomalías biológicas que afecten el comportamiento. Cabe indicar que el objetivo del presente art. consiste analizar la violencia intrafamiliar en el Ecuador y su incidencia en la salud ante la vulneración de los derechos del presunto agresor, para ello se empleó un enfoque cualitativo sustentando en el método inductivo - deductivo a partir de la revisión bibliográfica de textos jurídicos, artículos científicos y libros.

Descriptores: Violencia; Familia; Agresión; Agresor; Derechos.

\section{ABSTRACT}

Intra-family violence as a social, legal and public health problem, violates the rights of the alleged aggressor, generating legal consequences, transgressing constitutionally recognized rights, as well as the principle of innocence. Ecuadorian legislation has established conducive means of defense to avoid committing acts of provocation since the purpose is to prevent the alleged culprit and his victim from relating, in addition the medical analysis of mental health status is unavoidable to know if there are biological anomalies that affect behavior. It should be noted that the objective of this art. It consists of analyzing intra-family violence in Ecuador and its impact on health in the face of the violation of the rights of the alleged aggressor, for which a qualitative approach was used, based on the inductive - $d$ eductive method based on the literature review of legal texts, scientific articles and books

Descriptors: Violence; Family; Aggression; Aggressor; Rigths.

\section{INTRODUCCIÓN}

El Derecho Constitucional es la base jurídica y los cimientos propiamente dichos de todos los Estados; de ahí se regula la estructura formal y la construcción del ordenamiento jurídico que sostiene y garantiza los derechos, deberes y obligaciones del Estado, la sociedad y la familia como tal, desprendiéndose de estos principios importantes la supremacía constitucional, el mismo que resulta incuestionable, de ahí nacen normas jurídicas nacionales y tratados internacionales, por lo que resulta claro entender que todo acto normativo jurídico debe tener siempre estrecha analogía con la constitución, es decir, cada acto de derecho público o privado, debe poseer 
conectividad con la primacía constitucional, sin dejar de observar y considerar la legalidad del resultado de cada uno de los hechos jurídicos, por lo tanto, al estar investidos de elementos jurídicamente validos se entiende que su efecto repercute en la protección integral de los Estados y sus entes reguladores en razón de su materia (Carranza, 2015).

Por esta razón, es necesario analizar un tema importante como la violencia intrafamiliar su repercusión en la vulneración de derechos fundamentales, desde una perspectiva constitucional. Al respecto, varios tratadistas en temas de violencia intrafamiliar entre ellos, Figueroa (2015) afirma que es difícil proteger y avalar los estrictos derechos humanos de la sociedad, por cuanto no existen garantías esenciales que cumplan con la formalidad de proteger a la familia y a sus miembros, siendo estos mismos doblemente afectados, considerando además la repercusión de costumbres ancestrales dentro de una fase de dogmatismo cultural que ha permitido que la violencia día a día desencadene en un fenómeno social, que ha aumentado trances familiares que relativamente como consecuencia general ha desencadenado a la desintegración total de las familias.

Bajo este contexto, las evaluaciones mundiales publicadas mediante informe presentado por la Organización Mundial de la Salud (Organización Mundial de la Salud, 2017) revelan que una de cada tres mujeres en el mundo (35\%) han sufrido violencia física y/o sexual por parte de su pareja y que alguna vez en su vida fueron abusadas por terceros. Este informe también afirma, que en el abuso sexual se presenta en familiares directos de las víctimas, el $30 \%$ de las víctimas manifiestan haber sido abusadas física y/o sexualmente por parte de su pareja. El agresor busca justificar su actuación a través de explicaciones que perturban la tranquilidad de los miembros de la familia, se considera que existen causas determinantes que provocan procederes de violencia, una de ellas es la diferencia en que se encuentran muchas de las veces las mujeres y los hombres, ya sea por temas laborales o económicos que son determinantes en nuestra sociedad (López, 2004). 
Las personas agresivas propiamente dichas que ejercen la violencia de la manera más severa, incluyendo con abusos psicológico y sexual se caracterizan por tener múltiples antecedentes penales, cometer delitos de índole sexual, tener problemas con el alcohol y las drogas, además presentan enfermedades mentales como desórdenes de personalidad, en ocasiones mantienen una predisposición genética fuerte hacia la agresividad, así como también, poseen traumas por abusos o abandonos vividos durante su infancia. Para ellos, la violencia es una conducta normal a la provocación recibida (Medina, 2002) en la gran mayoría de los casos la violencia física, requiere atención médica especializada; sin embargo, las victimas que han sufrido violencia física no solicitan esta ayuda médica necesaria debido a que tratan de ocultarlo para no ser objeto de compasión o burlas (Benavides y Puebla, 2018).

Ante la situación expuesta en los párrafos que antecede, en el presente trabajo se plantea como problema de investigación la vulneración de los derechos del presunto agresor en la violencia intrafamiliar, como consecuencia del amplio margen legal instaurado en el Ecuador tanto en la protección de la víctima como la criminalización al agresor. En consecuencia, el art. tiene por finalidad analizar la violencia intrafamiliar en el Ecuador y su incidencia en la salud ante la vulneración de los derechos del presunto agresor.

\section{DESARROLLO}

\section{Un enfoque integral de la violencia intrafamiliar}

La historia mundial a través de sus aportes en el tema de violencia de género indica la multiplicidad de formas en que se vulnera no solo a la mujer sino a la humanidad, la coexistencia de factores exógenos en determinados casos, confluyen para que malas conductas o comportamientos aumenten y se precisan al enfoque del desarrollo del ser humano desde su infancia hasta su vejez (Briceño y Zubillaga, 2001). De esta manera el ejercicio de la violencia intrafamiliar constituye un fenómeno que resulta invisible a los ojos del agresor de tal manera que se determina esencialmente la forma de resolver un 
conflicto mediante el empleo de la fuerza, ya sea de modo físico o psicológico, para crear 0 mantener un desequilibrio de poder entre las partes, permanente 0 momentáneo.

En el caso de la violencia intrafamiliar, la absoluta predominancia de los varones en su ejercicio muestra que existe un conjunto de prácticas, símbolos, representaciones, normas y valores que las sociedades elaboran a partir de la diferencia sexual y que asigna posiciones jerarquizadas a varones y mujeres. Este tipo de organización social, denominada sistema de sexo/género, se caracteriza por la hegemonía que los varones poseen sobre las mujeres, dominio que también recibe el nombre de patriarcado (Álvarez, 2007).

Para entender cómo funciona y se ejerce la violencia intrafamiliar, es necesario conocer su definición, la cual se expone a continuación: la violencia intrafamiliar, de acuerdo a la OMS (2017), es definida como "aquella acción ejercida por una persona miembro de una familia que ejerce contra la persona más débil causándole daño físico o psíquico" (pág. 50). La violencia es la forma como se ejecuta el maltrato, donde se manifiestan sentimientos como odio, decepción, frustración, el desamor a otra persona, y que por lo general es la pareja del agresor, se puede decir que también la violencia intrafamiliar, es conocida con varios nombres como, por ejemplo: violencia familiar o violencia doméstica, sin embargo, todas se refieren al maltrato que recibe una persona por parte de otra. Estos términos son empleados en los diversos países según su cultura y costumbre.

Los componentes que originan la violencia intrafamiliar son diversos y pueden presentarse a cualquier edad reflejándose en la etapa adulta. La intolerancia es uno de esos factores debido a que las personas no controlan sus impulsos, al no poder dominarlos, actúan violentamente contra otras personas, en especial con sus parejas, la persona violenta no piensa o razona las circunstancias, reacciona de forma ofensiva con los miembros de su núcleo familiar, es decir, cuando está dominada por los celos 
se imagina cosas que no son, como las considera verdaderas agrede sin medir las consecuencias (Colina \& Ramírez, 2018).

La falta de vínculos afectivos es otro factor originario de la violencia, puesto que las personas que no han recibido cariño, comprensión y atención desde su infancia, no son capaces de demostrar estos sentimientos, son consideradas así por su falta afecto, por tal motivo adoptan conductas agresivas, poseen dificultad para resolver sus problemas. Debido a su baja autoestima, pierden toda noción de reacción, y ante una crisis o dificultad recurren a otros medios para evadirlos (Figueroa, 2015).

La violencia intrafamiliar tiene su origen en factores biológicos, como resultado de vivencias de episodios violentos en el hogar, el entorno socioeconómico, educacional y familiar que tuvo la persona en su infancia y antes de su etapa adulta. A través del estudio general de estos factores, se analiza cuál es su estructura familiar, dinámica y la crianza que determinan los antecedentes de violencia en el mismo; el entorno social es importante en el desarrollo del individuo de ahí se determinan procederes de desigualdad que influyen en el comportamiento dentro del convivir del hogar (Buvinic, Morrison, y Orlando, 2005).

Dentro de la violencia que se ejerce existen varios tipos que pueden ser ejecutados a través de la violencia doméstica, estos tipos son:

Violencia Sexual: es obligar bajo cualquier medio a la víctima a tener relaciones sexuales, en este caso no existe el placer sexual o está mezclado con sentimientos de culpabilidad (Silva, 2012) actuares que dejan secuelas de las cuales las afectaciones psicológicas deben ser tratadas por profesionales.

Violencia Física: se determina como el mal uso de la fuerza por parte del agresor frente a su víctima, en algunos casos deja como secuelas, lesiones graves y que necesitan tiempo para su recuperación, en el peor de los casos la víctima fallece debido a que no recibió ni siquiera apoyo inmediato de sus familiares por temor a recibir amenazas, golpes y enfrentamientos posteriores como producto de una venganza. Esta violencia 
es, junto a la sexual, la más reportada por las víctimas, de tal manera causan afectación y perturbación emocional al entorno familiar (Aldana Zavala \& Valles González, 2018). Violencia psicológica: constituye toda acción u omisión realizada que cause daño, dolor, perturbación emocional, alteración psicológica o disminución de la autoestima de la mujer o familiar agredido (Ibídem). Este tipo de violencia hace que la mujer, o la víctima, cambie su estado de ánimo y personalidad, además de crear sentimientos o pensamientos que no son ciertos, como, por ejemplo, el sentimiento de culpa o fracaso, provocando que realice acciones como, por ejemplo, alejarse de su familia y entorno afectivo, renunciar a su trabajo, transformar su forma de vestir y apariencia física, cambios de actitud, entre otros.

Violencia económica: la violencia económica afecta la estabilidad de otra persona. Se puede manifestar mediante limitaciones para controlar el ingreso obtenido, vigilar en que se destina el dinero o los gastos del hogar, revisar o manejar las cuentas bancarias; así como la percepción de un salario más bajo por igual trabajo, dentro de un mismo centro de trabajo, ya que la violencia económica se puede dar tanto a nivel de hogar como a nivel laboral. Esta forma de violencia que tiene que ver con la eliminación de la libertad financiera, trayendo como consecuencia un daño económico que afecta la sobrevivencia humana. Al igual que las demás formas de violencia, busca generar subordinación y temor de la víctima hacia el agresor estableciendo un patrón de desigualdad de género que se prolonga gracias a la violencia (Procuraduria General de Mexico, 2017).

\section{Perfil del agresor de violencia intrafamiliar}

En cuanto al agresor, existen varios conceptos, sin embargo, por lo general se define como aquella persona que ejerce violencia sobre otra persona, en este caso es su grupo familiar donde busca manifestar su frustración o depresiones a través de realización de actos violentos (Pérez, 2016). Todo acto de violencia que no persigue un propósito constitucional tiene que ser radicalmente castigado, sin embargo, a la 
justicia en la sociedad contemporánea no le interesa en muchos de los casos atender estos problemas, ya que se encuentran involucrados compromisos que merecen más atención (Cabanellas, 2007).

Es fácil reconocer las características del agresor y dependiendo de la circunstancia este reacciona de forma agresiva, por lo tanto, como lo califica Ramos (1994) son "Personas mentalmente desequilibradas y físicamente reconocibles por sus siniestras facciones". (pág. 79). Estas personas se caracterizan por tener una reacción desmedida y actúan de forma agresiva causando daño a su entorno y haciendo que sean conductas inadaptadas y antisociales. Por su parte, Briceño (2007) con el mismo criterio, considera que la violencia no es un hecho concreto o puntual, en donde existe una víctima o victimario; sino más bien se enmarca en las etapas anteriores y posteriores al momento de agredir a la víctima, debido a los factores que pueden generarla, como alcoholismo, drogas, la pobreza, la desigualdad, los rasgos culturales, celos, decepción, depresión, entre otros.

Para los autores Hamberger y Hastings (1986), existen entre los agresores tres tipos de personalidad: el Narcisista-Antisocial, el Esquizoide-Borderline y el Pasivo/dependienteCompulsivo, donde comparando grupos de individuos que agredieron en algún momento a sus parejas con otros que no lo habían hecho, encontraron estas características de personalidad: hostilidad con las mujeres, baja socialización, consumo de drogas, comportamiento agresivo, conducción peligrosa de vehículos, conducta delictiva y tendencias antisociales. El grupo de agresores resultó ser rígido, estereotipado y con dificultad para desarrollar relaciones reciprocas debido a su hipermasculinidad ${ }^{1}$ a través de las conductas típicas en el comportamiento del hombre en las relaciones interpersonales.

\footnotetext{
${ }^{1}$ La hipermasculinidad es la constante necesidad de querer demostrar la hombría y superioridad en relación al género femenino. La definición de hipermasculinidad se denomina a través de los estereotipos que son establecidos por la sociedad. García (2009) afirma que culturalmente lo masculino se identifica con lo rudo, con la agresividad, con la fuerza, con la potencia y la actividad.
} 
Un factor importante en la conducta agresiva de la persona es el aspecto económico. Desde el punto de vista de los autores Ávila, González y Rico (2015), a través de estudios biográficos de personas que han contado sus experiencias, afirman que su infancia fue vivida en medio de violencia, pobreza, abuso y alcoholismo, que ponen en contexto el hecho de que se repita ese patrón de conducta violenta en su etapa adulta, y en el caso de las mujeres, vivir con la experiencia de haber sufrido el maltrato y violencia con sus madres, hermanas y ellas mismas. En la misma línea de pensamiento, se expresa Ramírez (2002), quien en su investigación determina que a pesar de que el nivel socioeconómico no es factor directo en la violencia, el estilo de vida que llevaron las personas si favoreció la aparición de este fenómeno, por cuanto sintieron discriminación por su clase social, vivir el abandono del padre o su alcoholismo, tuvieron que dejar los estudios para trabajar y ser cabeza de hogar desde temprana edad.

\section{Base legal contra la violencia intrafamiliar en Ecuador}

En Ecuador existe un amplio margen legal tanto en la protección de la víctima de violencia intrafamiliar como la criminalización al agresor, aunque hay que dejar claro que ambas partes involucradas tienen derechos que deben ser protegidos, por lo que en esta investigación se busca determinar cuáles son las normas que lo protegen. Dentro de las bases jurídicas que se manejan para la atención de violencia intrafamiliar existe: La Constitución de la República del Ecuador, como norma suprema rectora, el Código Orgánico Integral Penal, como norma encargada de la judicialización y la determinación de las penas y la Ley Orgánica Integral para la Prevención, Erradicación de la Violencia de Género Contra Las Mujeres, como norma especializada en el tema, tratados y convenio internacionales para la protección de las personas víctimas de violencia.

La Constitución de la República del Ecuador, dentro de su normativa jurídica constitucional establece en el Art. 67.- Que la familia es la base fundamental de la 
sociedad, por lo tanto, debe ser protegida dentro de un ambiente sano en todos los aspectos, con armonía, respeto y tolerancia, al igual que el derecho a la vida, el cual es fundamental y que es determinante en los casos de femicidio, como máxima expresión de la violencia intrafamiliar (Asamblea Constituyente, 2008). Por otro lado, en el caso del agresor, habla sobre el respeto al debido proceso, con su doble función de derecho y principio procesal con todos los elementos que conlleva su aplicación, como es el ser juzgado por un juez de violencia intrafamiliar, tener la presencia de su abogado, garantizarle sus derechos durante el proceso, la presunción de inocencia, entre otros. Con la vigencia del Código Orgánico Integral Penal (COIP) ecuatoriano en el año 2014, se estableció de forma específica los tipos de violencia y sus respectivas sanciones de acuerdo a la gravedad de acción cometida por el presunto agresor, donde determina que debe existir proporcionalidad en la sanción que se aplica al infractor de la ley, fundamentalmente debe existir motivación jurídica en la resoluciones y sentencias.

En el caso de la violencia intrafamiliar, establecida en el COIP art. del 155 al 159, dentro de las medidas de protección que puede solicitar una persona que ha sido víctima de violencia está el requerimiento de la boleta de auxilio con la que se protege a la presunta víctima y la familia, esta medida trae como consecuencia la prohibición de acercamiento del presunto agresor, en este caso la afectada acude a solicitar ante la autoridad competente protección inmediata y se la otorgan sin comprobar efectivamente como fueron los hechos, ya que para la legislación penal la sola versión ya constituye prueba, sin considerar los derechos de quien en ese momento esta denunciado y que en algunos casos ni siquiera ha mantenido contacto con la denunciante, desde ese instante ya sus derechos son transgredidos en virtud de las consecuencias que conllevan la emisión de una boleta de auxilio (Código Orgánico Integral Penal, 2014).

Las medidas de protección establecidas en el art. 558 del COIP, están orientadas a proteger a las víctimas en casos de violencia contra la mujer y miembros del núcleo familiar, los objetivos de estas medidas de protección en lo que se refiere individualmente a cada una de ellas determinan que al presunto agresor se le prohíbe 
asistir a lugares específicos, con la finalidad de proteger a la víctima, Se le prohíbe aproximarse a la víctima o testigos en los lugares donde ellos se encuentren, para evitar que sean intimidados por el presunto agresor con la finalidad de dar seguridad a las mismas, no realizar persecución alguna peor aún intimidar a los miembros de la familia o la víctima por terceras personas, para de esta forma evitar la violencia en contra de la mujer o miembros del núcleo familiar (COIP, 2014).

En el caso de la boleta de auxilio la autoridad competente debe protegerle a la víctima con esta medida, con la finalidad de que el presunto agresor no cometa esta clase de contravención y no sea privado de su libertad. La Policía Nacional debe prestar auxilio de inmediato a la víctima y así evitar consecuencias nefastas en el futuro, se dispone la salida inmediata de la casa, con la única finalidad de dar protección a la víctima en lo que tiene que ver con la integridad física, sexual, e impedir de esta forma sea maltratada en forma verbal o golpeada por el presunto agresor, además de las medidas cautelares y de protección la autoridad competente tendrá la facultad de fijar una pensión para la subsistencia de la víctima que ha sido agredida por el presunto agresor, de acuerdo a la normativa vigente de la materia por lo que son medidas que cuidan la integridad personal de las víctimas y evitar a futuro los maltratos físicos, psicológicos y sexuales que son protegidos por la Constitución de la República del Ecuador, como por los Derechos Humanos y los Tratados Internacionales.

La Ley Orgánica Integral para la Prevención, Erradicación de la Violencia de Género Contra Las Mujeres ( Ley Orgánica Integral para Prevenir y erradicar la Violencia contra las Mujeres, 2018), es una norma relativamente nueva, que tiene como finalidad manejar todo lo relacionado con los casos de violencia. En ella se expone las competencias que tienen todos los organismos con los que se trabaja en el tema y los procedimientos a seguir cuando se presenta un caso. Por otro lado, busca ser también un instrumento de prevención y concientización para lograr la protección de la familia como institución, también para la transformación positiva de los patrones socioculturales existentes actualmente en la sociedad; y, a la generación de nuevas 
masculinidades, así como también brindar atención integral y protección oportuna a la persona que ha sido víctima.

Existen acuerdos y tratados internacionales que están enfocados a la protección integral de las víctimas de violencia, el Ecuador como Estado parte y signatario, debe cumplir y velar sin causa que lo límite para contrarrestar los índices de violencia, contribuir y ser referente mundial. Dentro de estos tratados se encuentran la Convención Sobre la Eliminación de Todas las Formas de Discriminación contra la Mujer, su misión está dirigida a contribuir para que lo índices de violencia mundial y la tortura criminal hacia la mujer sea cada año menor, es decir, que las estadísticas informen al mundo respecto de menos femicidios. Así mismo, existen países que han firmado su compromiso y al ser parte de esta convención, su obligación es contribuir a través de normativas jurídicas que vayan encaminadas y ligadas a la protección integral del ser humano en todo su contexto desde la concepción, sus legislaciones por supuesto tienen el propósito vinculante de garantizar que los derechos sean armónicos y recíprocos (Organizacion de las Naciones Unidas [ONU], 2011).

Por su parte, la Asamblea General de las Naciones Unidas, redactó la Declaración sobre la eliminación de la violencia contra la mujer, en donde se insta a los Estados a tomar medidas para hacer cumplir la ley y que los funcionarios que se encarguen de la prevención, investigación y castigo de la violencia contra la mujer se capaciten en cuanto a las necesidades de la mujer (ONU, 2011).

\section{Derechos del presunto agresor de violencia intrafamiliar}

El Estado está obligado a garantizar el bienestar, cuidado y protección en todas sus formas a todos sus ciudadanos, mucho más cuando estos están inmersos en un problema o son parte de un proceso judicial en su contra; para estos casos la ley les protege y constitucionalmente les brinda la oportunidad de defenderse de forma efectiva; tienen una posibilidad visible y real para hacer valer sus argumentos y razones ante el juzgador sustanciador del proceso judicial. 
Al establecer cuáles son los derechos que se vulneran al presunto agresor una vez que es denunciado por una supuesta víctima de violencia intrafamiliar, este es sujeto de derechos, por lo tanto se debe garantizar los mismos durante el proceso judicial mientras no se pruebe lo contrario, sobre todo en los casos en los que no pueda comprobarse que fue el agresor, se considera que se está prejuzgando sin pruebas, sin conocer los hechos en su contexto, por lo que se transgreden derechos constitucionales, tal es el caso de las garantías básicas al debido proceso entre las que la presunción de inocencia se deja en entre dicho ya que nadie puede ser declarado culpable, sino hasta que la autoridad sustanciadora de un proceso judicial penal o de violencia intrafamiliar la lo haya determinado y en base al aporte de prueba testimonial, material, pericial con la que haya aportado la persona que se cree afectada 0 vulnerada. (Asamblea Nacional Constituyente, 2008).

La indefensión es un precepto jurídico, que se refiere exclusivamente a la situación procesal de una persona en su derecho a defenderse y que no le permite intervenir de forma eficaz en la defensa de su interés y garantías legales dentro de un proceso jurídico. La situación en la que se encuentra la persona que no ha podido defenderse de forma correcta, ni hacer valer sus derechos ya que se configura indefensión. En consecuencia se afecta una garantía constitucional, debido a que en varias legislaciones se considera como un derecho estrictamente constitucional y que se vuelve fundamental dentro de un proceso penal ya que este permite tener un medio de defensa e inclusive designar un defensor público en caso de que el procesado o imputado no posea los recurso económicos necesarios para contratar a un abogado privado, la autoridad no podrá realizar un juzgamiento si el procesado no tiene un defensor que intervenga por sus derechos y garantías establecidos en la norma constitucional y otras leyes.

Otro derecho vulnerado es la Familia establecido en la Constitución de la Republica, art. 51 en donde reconoce a las personas privadas de la libertad los siguientes derechos. 2. La comunicación y visita de sus familiares y profesionales del derecho. El compromiso 
del Estado, sociedad y familia confluyen en la rehabilitación y reinserción de quienes son juzgados, a fin de que en lo posterior puedan llevar una vida organizada y con buenas prácticas, considerando que cada uno de los miembros de la familia necesitan recurrir a rehabilitación y terapias. (Asamblea Nacional Constituyente, 2008).

Por otro lado, el derecho a vivir dignamente se encuentra garantizado en la Constitución en su art. 30,donde establece que "Las personas tienen derecho a un hábitat seguro y saludable, y a una vivienda adecuada y digna, con independencia de su situación social y económica" (Ibídem, 2008, pág. 28), tanto la víctima como el agresor tienen derecho a vivir en un ambiente y entorno de paz, entendiendo por esto que en caso de violencia se debe optar por la separación de la pareja, sea esta por decisión de las partes afectadas o por medio del dictamen de un Juez en caso de haber una denuncia o un procedimiento judicial instaurado.

Además, son vulnerados los derechos económicos: mientras el presunto agresor no se encuentre inmerso en un proceso judicial, siempre y cuando la ley se lo permita, tiene derecho al trabajo, a recibir salarios, beneficios, realizar transacciones comerciales, disponer de dinero, entre otros, que la ley le faculte, para poder desarrollarse como ser humano sujeto de derechos, considerando que al tener que abandonar su casa por una mal infundada denuncia tiene que buscar un nuevo lugar para sobrevivir, aumentado de esta manera los gastos que conllevan su manutención fuera de su hogar.

Amas de estos, existen otros elementos que se vulneran cuando el presunto agresor es procesado judicialmente. En los casos donde se emplea la boleta de auxilio, que como se dijo en líneas anteriores, en muchos casos se solicitan sin realizar una investigación rigurosa del argumento (Ver figura 1), pierde el derecho a tener un hogar estable debido a que tiene que salir de su casa, lo que también implica la pérdida del derecho a la convivencia y a ejecutar sus deberes como padre. Otro aspecto importante conforme lo señala Sarquis, (2005) es que durante todo el proceso que conlleva el tratamiento de un caso de violencia intrafamiliar, no se habla de la afectación a la salud tanto física como psicológica del presunto agresor de violencia intrafamiliar, ya que siendo o no 
responsable, solamente con el hecho de saber que ha sido denunciado, es suficiente para que se ocasionen cambios de actitud en esa persona, puede sufrir un infarto, derrame cerebral, estrés, crisis hipertensiva, entre otros. A nivel psicológico se pueden presentar cuadros de ansiedad, depresión, y angustia.

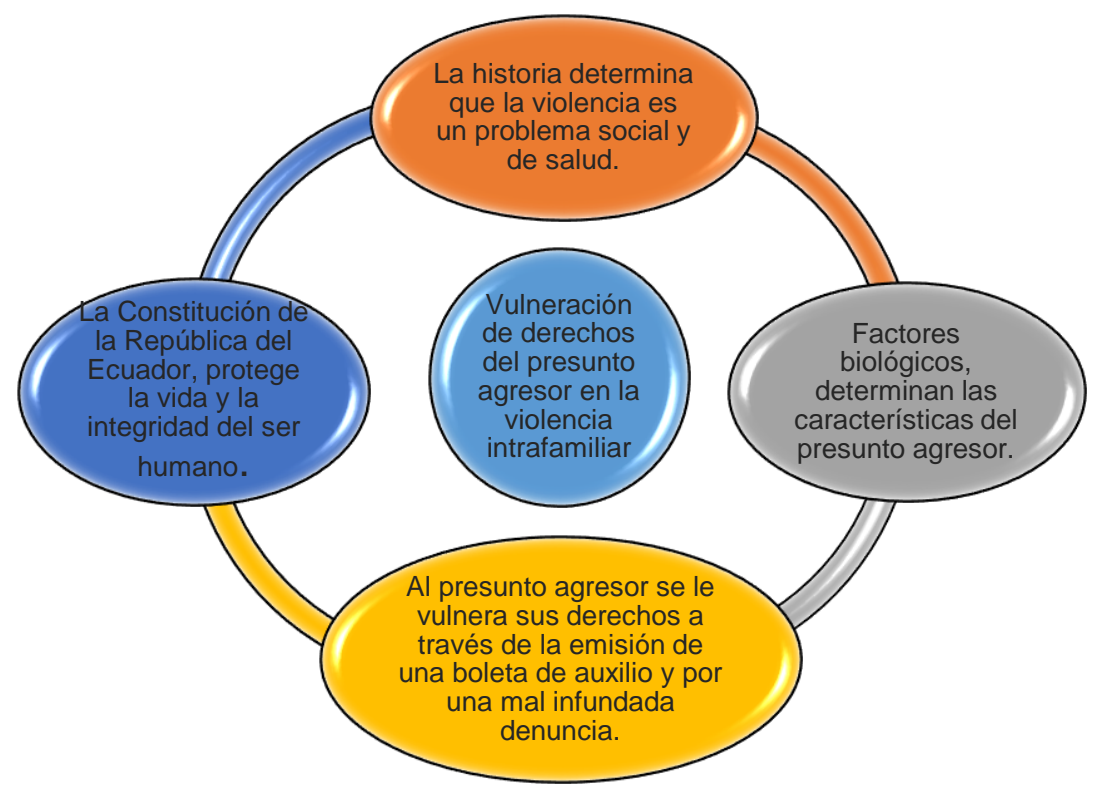

Figura 1. Vulneración de derechos del presunto agresor de violencia familiar

\section{METODOLOGÍA}

Para esta investigación, se realizó la revisión exhaustiva de bibliografía, diccionarios jurídicos, doctrina y artículos referentes a los diversos temas tratados, desde un enfoque integral de la violencia intrafamiliar, para determinar que el objetivo propuesto cumple con el análisis doctrinario que admite la descripción sistemática y homogénea del fenómeno que se está estudiando, con la finalidad de conocer algunos aspectos de los cuales se pretende tener respuesta a través de un conocimiento más profundo para sustentar el art. de revisión.

A través del análisis cualitativo y de la investigación de las variables, se obtuvo información precisa la misma que permitió entender que el presunto agresor en temas de violencia intrafamiliar es constitucionalmente vulnerado, ya que en la aplicación del 
método deductivo se concluye que la supuesta víctima es protegida sin ni siquiera haber probado su daño o afectación (Padilla, Erazo y Narváez, 2019).

\section{APORTES}

La violencia intrafamiliar es un flagelo que afecta a la sociedad y que tiene su origen en muchos factores que son intrínsecos y externos de las personas, sin embargo, esos elementos no le dan derecho a ningún individuo a agredir a otro.

Por otro lado, en la investigación se determina que la agresividad es uno de los elementos más importantes que forman parte de la violencia, y que además tiene varios factores sociales, biológicos, culturales y psicológicos que afectan las relaciones humanas y sentimentales del ser humano ocasionando que actúe violentamente.

Se evidencia que no hay un perfil único para identificar a un agresor, por cuanto cada persona y las circunstancias en la que se da la violencia son diferentes, sin embargo, también es cierto que en el comportamiento del agresor existen orígenes y modos de actuar que pueden determinar su carácter agresivo y las consecuencias que se generan.

En el sistema penal ecuatoriano no se cumple el principio de igualdad procesal para las partes en los casos de violencia intrafamiliar, por lo que al presunto agresor se le vulneran los principios y derechos constitucionales, la sola versión de la presunta víctima es suficiente para considerar que su vida corre peligro y que se debe disponer que su pareja o presunto victimario salga de la casa en donde conviven con sus hijos, dejándolo en absoluta indefensión.

Se concluye que además de garantizar los derechos tanto humanos como procesales al agresor, también es necesario que se cuide su estado físico y psicológico, ya que determina su estado emocional y su salud, no obstante, es obligación del Estado, sociedad y familia brindar el acompañamiento a la víctima en su momento a sus hijos y al victimario para su reinserción en la sociedad como entes productivos. 


\section{REFERENCIAS CONSULTADAS}

1. Aldana Zavala, J., \& Valles González, E. (2018). La mujer en Derecho a una Vida libre de violencia desde la Organización Comunitaria. IUSTITIA SOCIALIS, 3(5), 110-127. doi:http://dx.doi.org/10.35381/racji.v3i5.171

2. Álvarez, F. (2007). Los varones frente a la ley de violencia intrafamiliar: una perspectiva de género. Revista juridica regional y subregional andina 7, 104.

3. Asamblea Constituyente. (2008). Constitución de la República del Ecuador. Montecristi, Ecuador.

4. Asamblea Nacional Constituyente. (2008). Constituciòn de la Repùblica del Ecuador. Montecristi.

5. Ávila, L., González, E., y Rico, A. (2015). Enfoque biográfico-narrativo para comprender la violencia intrafamiliar. Mèxico.

6. Benavides, B., y Puebla, C. (2018). La falta de una disposición legal que determine en que casos debe revocarse las medidas de protección del Código Orgánico Integral Penal. Quito. Obtenido de http://www.dspace.uce.edu.ec/handle/25000/15644

7. Briceño, R. (2007). Sociología de la violencia. Quito.

8. Briceño, R., y Zubillaga, V. (2001). Dimensiones y Construcciones de la Violencia en América Latina. Caracas, VENEZUELA: LACSO.

9. Buvinic, M., Morrison, A., y Orlando, M. (2005). Violencia Crimen y Desarrollo. México.

10. Cabanellas, J. (2007). Diccionario Enciclopedico de Derecho Usual. Buenos Aires, Argentina: Heliasta S.R.

11. Carranza, J. (2015). Temas de Derecho Constitucional.

12. Código Orgánico Integral Penal. (2014). Recuperado de https://www.defensa.gob.ec/wpcontent/uploads/downloads/2018/03/COIP_feb2018.pdf

13. Colina R., L., \& Ramírez R., M. (2018). Promoción de los Derechos de la Mujer a una vida libre de violencia a través de un Comité pro defensa. IUSTITIA SOCIALIS, 3(4), 60-83. $\quad$ Recuperado de 
https://fundacionkoinonia.com.ve/ojs/index.php/lustitia Socialis/article/view/122/1 $\underline{04}$

14. Figueroa, B. (2015). Teoría y práctica sobre la violencia contra la mujer y miembros del nucleo familiar. 2. Quito, Ecuador: Ofigraf.

15. Hamberger, k., y Hastings, J. (1986). Personality Correlates of Men Who Abuse Their Partners: A Cross-Validation Study 1. Londres.

16. Ley Orgánica Integral para Prevenir y Erradicar la Violencia contra las Mujeres. (2018). Quito.

17. López, E. (2004). La figura del agresor en la violencia de género: características personales e intervención. Marid.

18. Medina, J. (2002). Violencia contra la mujer en la pareja: investigación comparada. Valencia.

19. ONU. (30 de 12 de 2011). Principales leyes, instrumentos y acuerdos internacionales $y \quad$ regionales. Obtenido de https://www.endvawnow.org/es/articles/1125-principales-leyes-instrumentos-yacuerdos-internacionales-y-regionales.html

20. Organización Mundial de la Salud. (29 de 11 de 2017). Violencia contra la mujer. Obtenido de http://www.who.int/es/news-room/fact-sheets/detail/violence-againstwomen

21.Padilla Campoverde, D. A., Erazo Álvarez, J. C., \& Narváez Zurita, C. I. (2019). Diagnóstico situacional del potencial turístico de la parroquia Ricaurte, Azuay. Koinonía, 385

22. Pérez, M. (20 de 3 de 2016). LA VIOLENCIA INTRAFAMILIAR. Obtenido de https://revistas.juridicas.unam.mx/index.php/derechocomparado/article/view/3595/4336

23. Procuraduría General de México. (11 de 06 de 2017). Definición de Violencia económica. México. Obtenido de https://www.gob.mx/cms/uploads/attachment/file/242427/6_Enterate_Violencia_ econo_mica_y_patrimonial_contra_las_mujeres_junio_170617.pdf

24. Puebla, S. (2018). La falta de una disposición legal que determine en que casos debe revocarse las medidas de protección del Código Orgánico Integral Penal. Quito. 
25. Ramírez, M. (2002). Hombres violentos. Un estudio antropológico de la violencia. Mèxico.

26. Ramos, P. (1994). Conducta interpersonal: Ensayo de Psicología Social sistemática. Salamanca. Salamanca.

27. Sarquis, C. (2005). Introduccion al Estudio de la Pareja Humana. Santiago, chile: Universidad Católica de Chile.

28. Silva, V. (30 de 11 de 2012). Violencia Intrafamiliar. Revista Medica Junta de Beneficiencia de Guayaquil, 20. Obtenido de https://www.institutoneurociencias.med.ec/component/k2/item/851-violenciaintrafamiliar. 